ALEA, Lat. Am. J. Probab. Math. Stat. 16, 711-728 (2019)

DOI: $10.30757 /$ ALEA.v16-25

\title{
How sharp are classical approximations for statistical applications?
}

\author{
Jean-Marc Azaïs and Stéphane Mourareau \\ Institut de Mathématiques de Toulouse, Université Paul Sabatier \\ 118 route de Narbonne \\ 31062 Toulouse, France \\ E-mail address: jean-marc.azais@math.univ-toulouse.fr \\ Institut de Mathématiques de Toulouse, Université Paul Sabatier \\ 118 route de Narbonne \\ 31062 Toulouse, France \\ E-mail address: stephane.mourareau@gmail.com
}

\begin{abstract}
This paper aims at comparing theoretical approximations of the tail of the maximum of stochastic processes and the corresponding numerical evaluations. More particularly, we focus on the Pickands or double sum method, the Rice method, the Euler Characteristic method and a new one called the Poisson method. The numerical evaluation, performed using mainly Quasi Monte-Carlo integration and adaptations of the programs of Genz, show the domains of validity of each method.
\end{abstract}

\section{Introduction}

The distribution of the maximum of a Gaussian vector, process or fields has many application in Statistics and Spatial Modeling (see Worsley et al., 1996 or Cressie, 2015). For instance, in the "signal plus noise model" on the real line, we assume that the observation $\mathcal{Y}=(Y(t))_{t}$ is the sum of a signal $\mu=(\mu(t))_{t}$ and a noise $\chi=(X(t))_{t}$ which is a centered stationary Gaussian process. If we want to test the null hypothesis $\mathbb{H}_{0}: \mu \equiv 0$, a natural statistic is

$$
M_{T}=\max _{t \in[0, T]} X(t)
$$

the observed maximum over the interval $[0, T]$. Note that the maximum of the absolute value is also a relevant statistics, but for the sake of simplicity, we will not consider this last case which is very similar. Computing thresholds and power in our case involve some computations on the distribution of $M_{T}$. Unfortunately, it is

Received by the editors September 6th, 2018; accepted April 18th, 2019.

2010 Mathematics Subject Classification. Primary: 60G15, 60-04, secondary: 62-04.

Key words and phrases. Gaussian Process, Kac-Rice formula, Pickands method, Euler characteristic method. 
a largely unsolved problem and exact closed formulas exist only in some particular cases. In the other cases, some bound or asymptotic expansions have been constructed starting from the pioneering work of Rice (1944) then Pickands III (1969) and Adler (1981).

These expansions have generally good properties when $T$ is fixed and $u$ is large $(u \rightarrow \infty)$, but in statistical applications as computation of 0.95 fractile for $M_{T}, T$ and $u$ are linked and both can be "rather large". In such a case nothing is known on the properties of the considered expansions but if we focus on the OrnsteinUhlenbeck process and if we consider the Pickands approximations given by

$$
\mathbb{P}\left\{M_{T}>u\right\} \simeq T u \times \frac{\exp \left(-u^{2} / 2\right)}{\sqrt{2 \pi}} \text { and } \mathbb{P}\left\{M_{T}>u\right\}=T u^{2} \times \mathbb{P}(\mathcal{N}(0,1)>u),
$$

a numerical study show a very interesting behaviour. Indeed, Figure 1.1 suggests that the Pickands approximations are sharp for large values of $T$ but unsuitable for small values of $T$. Moreover, in this example the first approximation is a little more precise than the second one but it is not always the case, see Section 4.4.1.
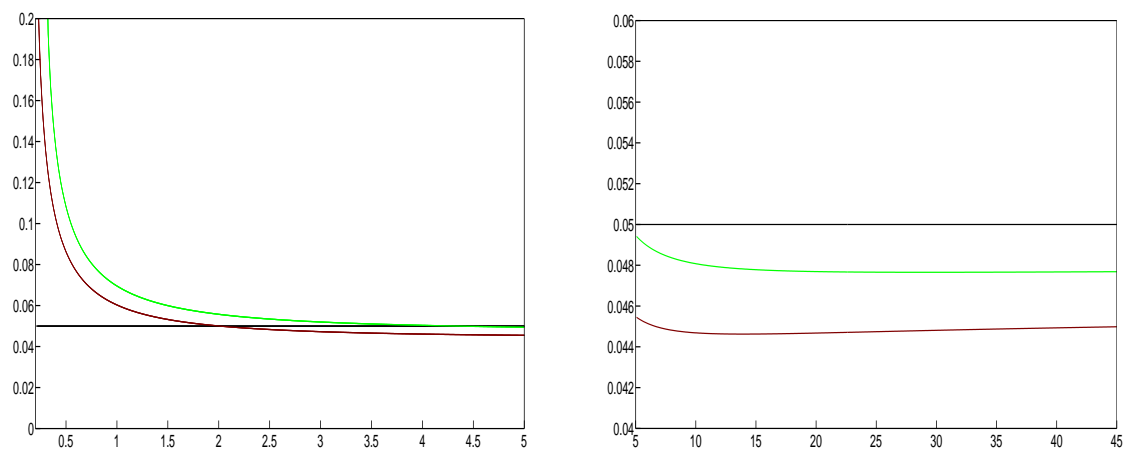

Figure 1.1. Exact level of a test constructed for a nominal level of $\alpha=\mathbf{5} \%$ using the Pickands method and the Ornstein-Uhlenbeck process. On the left: small intervals, on the right: large intervals (see Section 2). The level corresponding to equation (2.2) is given in brown while the one of equation (2.3) is given in green. Both are computed thanks to the pou program (see Section 3.1.1) and according to the procedure of the Section 4.1.

Furthermore, if we want to compute now the median of $M_{T}$, and if we use the Rice method defined in Section 2.2, Figure 1.2 shows that the method does not perform accurately for large values of $T$.

In light of the above examples, the main contribution of this paper will be threefold.

- In Section 2.3 we present a new approximation based on a Poisson approximation.

- In Section 3.2.1 and 3.2.2, we present an extension of the MGP program of Genz (see Azaiis and Genz, 2013), firstly to non-stationary case, secondly to random fields.

- In Section 4, we use these programs and MGP to address the quality of the above-mentioned expansions for two precise problems.

- Computing a critical value at level $\alpha$ for statistical purpose.

- Computing the median of the distribution of the maximum. 


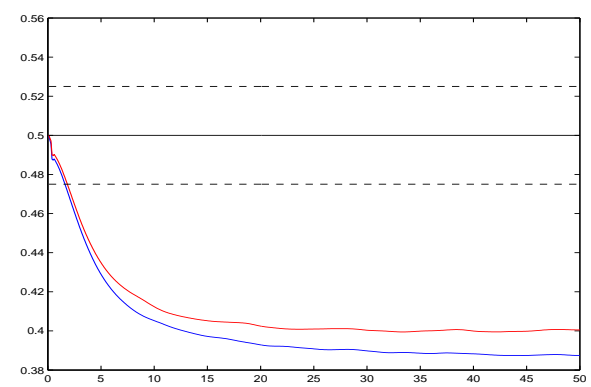

Figure 1.2. Exact probability to be above the approximate median computed using the Rice method (see Section 2). An upper bound (red line) and a lower bound (blue line) of the level are computed by the program MGP (see Section 3.1.1). The dotted lines indicate a relative variation of $50 \%$ with respect to the nominal level i.e. $47.5 \%$ and $52.5 \%$. The process considered has covariance function $\rho(t)=\exp \left(-t^{2} / 2\right)$.

As far as we know, it is the first time that such a numerical experiment is conducted.

In addition, Section 2 describes the approximations widely encountered in the literature as well as the new Poisson approximation. Section 3 describes the numerical methods that, except for those of Section 3.1, are modifications or improvements of the seminal work of Genz (1992).

\section{Description of the approximations}

We provide here a brief summary of approximation methods for $\mathbb{P}(M>u)$ where $M$ is the maximum of a Gaussian process or random field on sets detailed below. Before we introduce our assumption and notation.

- $\chi=(X(t))_{t}$ is a Gaussian process defined on $[0, T]$ or a Gaussian field defined on the rectangle $\left[0, T_{1}\right] \times\left[0, T_{2}\right]$ (excepting the Bessel process of Section 3.1.1). Its expectation is $m(t)$, its covariance is $r(s, t)$ in the general case and $\rho(h)=r(t, t+h)$ if $\chi$ is stationary.

- $M_{T}$ and $M_{T_{1}, T_{2}}$ are respectively the maximum of $X(t)$ on $[0, T]$ and $\left[0, T_{1}\right] \times$ $\left[0, T_{2}\right]$.

- $\phi$ and $\Phi$ denote respectively the probability density function and the cumulative distribution function of a standard normal variable. Moreover, set $\bar{\Phi}=1-\Phi$.

- In case of stationarity, we suppose that $\chi$ is centered and reduced, i.e. $\rho(0)=1$.

- In case of stationarity and differentiability, we suppose that $\chi$ is centered, reduced with unit speed, i.e. $\operatorname{Var}\left(X^{\prime}(t)\right)=-\rho^{\prime \prime}(0)=1$.

- $x^{+}=\max (x, 0)$ is the positive part of $x$.

- $p_{Z}(z)$ denotes the density of the random variable $Z$ at point $z$ when it exists.

2.1. Pickands method. Let $0<\beta \leq 2$ and $\chi$ be a $\beta$-process i.e. stationary Gaussian process with covariance function $\rho$ satisfying

$$
\rho(t)=1-C|t|^{\beta}+o\left(|t|^{\beta}\right) \text { as } t \rightarrow 0,
$$


where $C>0$, then the Pickands approximations, as $u$ tends to infinity, are given by

$$
\mathbb{P}\left\{M_{T}>u\right\} \simeq T C^{1 / \beta} u^{(2 / \beta)-1} \phi(u) H_{\beta},
$$

see Pickands III (1969), Piterbarg (1996) or

$$
\mathbb{P}\left\{M_{T}>u\right\} \simeq T C^{1 / \beta} u^{(2 / \beta)} \bar{\Phi}(u) H_{\beta},
$$

see Pickands III (1969), Albin and Choi (2010) or Michna (2017). More precisely, the $H_{\beta}$ are the Pickands constants and $T>0$.

The main drawback of the method is that the constants $H_{\beta}$ are known explicitly only in the cases $H_{1}=1$ and $H_{2}=(\pi)^{-1 / 2}$ (see Leadbetter et al., 1983 or Albin and Choi, 2010). In the other cases, some numerical approximation must be used as in Dieker and Yakir (2014) where the conjecture

$$
H_{\beta}=\left(\Gamma\left(\beta^{-1}\right)\right)^{-1},
$$

with $\Gamma$ the Gamma function, is discussed. Indeed, this conjecture has been proved to be false by Harper (2017) for small values of $\beta$.

In addition, the approximations given by (2.2) and (2.3) are obviously not efficient when $T$ is small. An heuristic improvement would be to add the factor $\bar{\Phi}(u)$ that appears in the Rice method hereunder leading to four versions of the Pickands approximation and raising the question:

Which method is the best one?

This question will be addressed in Section 4. Finally, note that the Pickands method, also called double sum method, has received a lot of generalization to various situations. The interested reader may consult Dębicki et al. (2016) and references therein.

2.2. Rice method. Let $\chi$ be a stationary Gaussian differentiable process then the Rice bound is given by

$$
\mathbb{P}\left\{M_{T}>u\right\} \leq \bar{\Phi}(u)+T \frac{\phi(u)}{\sqrt{2 \pi}}
$$

Note that both Rice and Pickands approximations apply ( the second with $C=\frac{1}{2}$ since we have assumed that $\left.\rho^{\prime \prime}(0)=-1\right)$ when $\beta=2$ in (2.1). In that case, Rice is asymptotically more precise as $u \rightarrow+\infty$ (see Azaïs and Wschebor, 2009, chapter 4). Moreover, the Rice bound, under some regularity conditions, is super-exponentially sharp in the sense that the error is bounded by

$$
C \exp \left(-\frac{u^{2}(1+\delta)}{2}\right)
$$

for some positive constants $C$ and $\delta>0$. Finally, there exists a non stationary version of formula (2.4) given by

$$
\mathbb{P}\left(M_{T}>u\right) \leq \mathbb{P}(X(0)>u)+\int_{0}^{T} \mathbb{E}\left(\left(X^{\prime}(t)\right)^{+} \mid X(t)=u\right) p_{X(t)}(u) d t,
$$

where the conditional expectation may be easily computed in the Gaussian case. 
2.3. Poisson method :A new approximation. Let us focus on stationary differentiable Gaussian processes. In that case, we already know that the Rice method, see Azaïs and Wschebor (2009), gives the upper bound (2.4). This bound is very efficient for small intervals and rather high levels. But of course it cannot be very accurate if $u$ is fixed and $T$ is very large, as it can be even larger than 1 , so, almost every time, a better approximation is given by using the Poisson Clamping heuristic of Aldous (1989). Basically, it assumes that the number $U_{u}(\chi,[0, T])$ of up-crossings of the level $u$ on $[0, T]$ follows a Poisson distribution so that the probability of having at least one crossing is approximatively $1-\exp \left(-\mathbb{E}\left(U_{u}(\chi,[0, T])\right)\right)$. Note that when $u$ and $T$ tend jointly to infinity so that $\mathbb{E}\left(U_{u}(\chi,[0, T])\right)$ is constant, the Volkonskii Rozanov theorem (see Volkonskil and Rozanov, 1959) implies that the limit is actually Poisson. Taking into account the fact that the limit as $T$ goes to 0 should be $\bar{\Phi}(u)$, we are conducted to use the approximation

$$
\mathbb{P}\left\{M_{T}>u\right\} \simeq \bar{\Phi}(u)+\Phi(u)\left(1-\exp \left(-\frac{T \phi(u)}{\sqrt{2 \pi} \Phi(u)}\right)\right) .
$$

Theoretically, a Taylor expansion shows that the bound from the Rice method and the Poisson approximation are equivalent when $u \rightarrow+\infty$. A discussion concerning it's numerical validity and performance is given in Section 4.

2.4. Euler characteristic method. The Euler Characteristic method is defined in its full generality for random fields defined on stratified manifold of $\mathbb{R}^{d}$ having constant variance (say equal to one). However, the formula is cumbersome and no numerical application exists. For simplicity we limit our attention to a particular case.

Let $\chi$ be a Gaussian centered differentiable isotropic random field on the rectangle $\left[0, T_{1}\right] \times\left[0, T_{2}\right]$. Denote $\rho(\|t-s\|)=\operatorname{Cov}(X(s), X(t))$ and suppose that $\chi$ is centered, reduced and with unit speed (this can always be obtained by a scaling) then the Euler characteristic approximation is given by

$$
\mathbb{P}\left\{M_{T_{1}, T_{2}}>u\right\} \simeq \bar{\Phi}(u)+\left(T_{1}+T_{2}\right) \frac{\phi(u)}{\sqrt{2 \pi}}+T_{1} T_{2} u \frac{\phi(u)}{2 \pi},
$$

see Adler and Taylor (2007) or Azaïs and Delmas (2002) for more details. In particular, the Euler Characteristic method is the natural generalization of the Rice method to dimension 2.

2.5. Other method. For completeness we must cite the work of Li and Xu (2018) which is dedicated to a method of importance sampling for rare events simulations applied to high values of random fields. Their method is very accurate for very high levels, typically $\mathbb{P}\left\{M_{T}>u\right\} \simeq 10^{-6}$ or $10^{-12}$. Since events we consider here have probability $0.5,0.05$ or 0.01 , we will not focus on thier method in the following.

\section{Numerical methods and programs}

In this section we present the juges of the numerical comparison of Section 4 .

3.1. Existing programs. The simplest cases are the few situations cases where the exact result is known by a closed formula and when this formula is simple enough so that a numerical program is available (the second condition being more restrictive than the first one). 
3.1.1. Exact results. Among the few classical cases where an explicit formula exists, see Azaïs and Lozada-Chang (2013), some are rather easy to compute and have been incorporated to the Maxgbbox toolbox More precisely, the following cases are covered:

- The Brownian motion or Wiener process : functions pbm, qbm, pnbm and qnbm.

- The Brownian bridge: pbb, qbb, pnbb, qnbb.

- The sine-cosine process: psc, qsc, pnsc and qnsc.

- The Ornstein-Uhlenbeck process: pou, qou, pnou, qnou.

- The Bessel process: pbp, qbp, pnbp, qnbp.

The prefix $\mathrm{p}$ is for the cumulative distribution, $\mathrm{q}$ is for the inverse distribution function or fractile function and $\mathrm{n}$ is for the norm or the absolute value.

3.1.2. A first approach for Gaussian vector. The first program QSIMVN written by Genz (1992) deals with integration of Gaussian densities over hyper-rectangles. It uses Quasi Monte-Carlo integration. In particular, considering an hyper rectangle in $\mathbb{R}^{n}$ which is the product $n$ times of $(-\infty, u)$, it allows us to compute numerically the distribution of the maximum of a Gaussian vector.

A complementary version named QSIMVNEF computes the expectation of a function with respect to the Gaussian density.

3.1.3. Record method and Quasi Montecarlo integration for Gaussian processes. The method relies on the record method (see Mercadier, 2006 and Azaïs and Wschebor, 2009) which is a refinement of the Rice method.

Theorem 3.1. Suppose that the process $\chi$ is Gaussian with $\mathcal{C}^{1}$ paths and satisfies:

- $\forall(s, t) \in[0, T]^{2}, s<t$, the distribution of $(X(s), X(t))$ does not degenerate,

- $\forall t \in[0, T]$, the distribution of $\left(X(t), X^{\prime}(t)\right)$ does not degenerate, then

$$
\mathbb{P}\left(M_{T}>u\right)=\mathbb{P}(X(0)>u)+\int_{0}^{T} \mathbb{E}\left(X^{\prime}(t)^{+} \mathbb{1}_{X(s) \leq u \forall s<t} \mid X(t)=u\right) p_{X(t)}(u) \mathrm{d} t .
$$

The formula (3.1) is in fact an implicit formula which has its own theoretical interest but is not directly usable for a numerical purpose. The condition $X(s) \leq u \forall s<t$ has to be discretized using a grid on the set $[0, T]$ giving now the following upper-bound:

$$
\mathbb{P}(X(0)>u)+\int_{0}^{T} \mathbb{E}\left(X^{\prime}(t)^{+} \mathbb{1}_{X(s) \leq u \forall s<t \in D_{n}(T)} \mid X(t)=u\right) p_{X(t)}(u) d t,
$$

where $D_{n}(T):=\left\{t_{0}=0, t_{1}=T / n, \ldots, t_{n}=T\right\}$ is, for example, the regular grid of $[0, T]$. To complete this study, a lower bound is obtained simply by discretization of time and using the routine QSIMVN of Section 3.1.2.

After the pioneering work of Mercadier (2006) with the toolbox MAGP, this method has been fully detailed in the program MGP written by Genz and described in Azaiis and Genz (2013). Note that MGP gives an upper bound (which is always displayed in red in the figures) and a lower bound (diplayed in blue). These bounds 
account for numerical errors, so in the examples displayed on Figures 1.2, 4.3, 4.4, $4.9,4.10$, it is possible to know in which interval the true probability lies.

In addition, in some cases, we have performed computer intensive studies improving the different parameters of MGP: number of points of the grid, number of QMC points. In such a case, in general, the upper bound and the lower bound converge to the same value which is the true value. Moreover, from these experiments we know that, in general, the upper-bound (in red) is more accurate than the lower bound (in blue).

\subsection{New programs.}

3.2.1. Record method for non stationary processes. We present here a generalization of MGP called MAXNSGP. At cost of being rather slower, it is adapted to noncentered non-stationary differentiable Gaussian processes.

This program provides an upper bound and a lower bound for the cumulative distribution function of the maximum. The lower bound is based on a time discretisation similar to the one of MGP. As for the upper bound, we consider the equation (3.2) and

- Use a trapezoidal rule to compute the integral using the same grid $D_{n}(T)$ so that we limit our attention to the computation of integrands at points $t_{i}=i / n, i \leq n$.

- For each $t_{i} \in D_{n}(T)$, set $Y=\left(X^{\prime}\left(t_{i}\right), X\left(t_{0}\right), \ldots, X\left(t_{i}\right)\right)=\left(Y_{1}, \ldots, Y_{i+2}\right)$ and note that

$$
\begin{gathered}
\left.\mathbb{E}\left(X^{\prime}\left(t_{i}\right)^{+} \mathbb{1}_{X(s) \leq u \forall s<t_{i} \in D_{n}(T)}\right) \mid X\left(t_{i}\right)=u\right) p_{X\left(t_{i}\right)}(u) \\
=\int_{0}^{+\infty} y_{1} \int_{]-\infty, u]^{i}} p_{Y}\left(y_{1}, \ldots, y_{i+1}, u\right) \mathrm{d} y_{1} \ldots \mathrm{d} y_{i+1},
\end{gathered}
$$

where $p_{Y}$ denotes the density of the Gaussian vector $Y$ and let $\Sigma_{i}$ be its variance-covariance matrix.

- Use the change of variable $Y=\Sigma_{i}^{1 / 2} Z$ where $\Sigma_{i}^{1 / 2}$ is the square root of $\Sigma_{i}$ obtained via the Cholesky decomposition. Note that $Z \sim \mathcal{N}\left(0, I_{i+2}\right)$.

- Use the change of variable $U_{j}=\Phi\left(Z_{j}\right)$ where the $U_{j}$ are independant and follow a uniform distribution for all choice of $j$.

- Reduce the problem to the hypercube $[0,1]^{i}$.

- Finally use MCQMC (Monte-Carlo Quasi Monte-Carlo) integration method on $[0,1]^{i}$. By this last method we mean that in general Quasi Monte-Carlo are very precise but do not give a reliable estimation of their error. To get this estimation we add an extra Monte-Carlo layer. See Genz (1992) and Azaïs and Genz (2013) for more details.

Contrary to MGP, the non stationarity of $\chi$ do not allow to construt recursively the numerous variance-covariance matrices $\Sigma_{i}$ as $i$ varies. By consequence, MAXNSGP is slower but more general. An exemple of application of MAXNSGP, available at Mourareau (2015), is given in Appendix A.

3.2.2. Record method in dimension 2. In dimension two, the record method is adapted to a Gaussian field defined on compact, convex subsets having a.s. a piecewise $\mathcal{C}^{1}$ parametrization of the boundary. Here we limit our presentation to the case 
where the parameter set is the rectangle $\left[0, T_{1}\right] \times\left[0, T_{2}\right]$. We use the lexicographic order defined by

$$
s=\left(s_{1}, s_{2}\right) \triangleleft\left(t_{1}, t_{2}\right)=t \Longleftrightarrow\left\{s_{2}<t_{2}\right\} \text { or }\left\{s_{2}=t_{2} ; s_{1}<t_{1}\right\}
$$

and define the record set similarly to the one dimensional case with the relation $\triangleleft$ instead of $<$. Our aim is to apply a Kac-Rice formula on the rectangle, which imply the application of Kac-Rice formula in dimension higher than 1. To do so, set $X_{1}^{\prime}=\frac{\partial X}{\partial t_{1}}$ and define the following mild hypotheses for $\chi$ :

(A1) The sample paths of the random field $Z=\left(X, X_{1}^{\prime}\right)$ are a.s. $\mathcal{C}^{1}$.

(A2) The distribution of $Z(t)$ does not degenerate.

(A3) For every $a \in \mathbb{R}^{2}, \mathbb{P}\left(\exists s \in\left[0, T_{1}\right] \times\left[0, T_{2}\right] ; Z(s)=a, \operatorname{det}\left(Z^{\prime}(s)\right)=0\right)=0$.

Sufficient conditions for $(A 1)-(A 3)$ are generic. They are almost always satisfied in the case of a process $\chi$ which is stationary. In that context, Mercadier (2006) proved the following theorem.

Theorem 3.2. With assumption above, for every $u \in \mathbb{R}$,

$$
\begin{aligned}
& \mathbb{P}\left(M_{T_{1}, T_{2}}>u\right)=\mathbb{P}(X(0)>u) \\
& +\sum_{i=1}^{3} \int_{B_{i}} \mathbb{E}\left(\left|X_{T}^{\prime}(t)\right| \mathbb{1}_{X(s)<X(t), \forall s \triangleleft t} \mid X(t)=u\right) p_{X(t)}(u) \mathrm{d} \sigma(t) \\
& +\int_{0}^{T_{1}} \int_{0}^{T_{2}} \mathbb{E}\left(X_{11}^{\prime \prime}-(t) X_{2}^{\prime+}(t) \mathbb{1}_{X(s)<X(t), \forall s \triangleleft t} \mid\left(X, X_{1}^{\prime}\right)(t)=(u, 0)\right) p_{X(t), X_{1}^{\prime}(t)}(u, 0) \mathrm{d} t,
\end{aligned}
$$

where

- $B_{1}=\left[(0,0),\left(T_{1}, 0\right)\right], B_{2}=\left[(0,0),\left(0, T_{2}\right)\right], B_{3}=\left[\left(T_{1}, 0\right),\left(T_{1}, T_{2}\right)\right]$ are the three relevant boundaries of the rectangle: the fourth one does not contribute because of the conditions.

- $\sigma$ is the surfacic measure (here the length) on the boundary.

- $X_{i}^{\prime}(t)=\frac{\partial X}{\partial t_{i}}, X_{11}^{\prime \prime}(t)=\frac{\partial^{2} X}{\partial t_{1}^{2}}$ and $X_{T}^{\prime}$ denote the derivative of $X$ along $B_{i}$ for $i=1,2,3$.

From formula (3.4), we can derive a numerical routine to bound $\mathbb{P}\left(M_{T_{1}, T_{2}}>\right.$ $u$ ) for non-stationary non-centered differentiable Gaussian fields. This program uses the same tricks than MAXNSGP, the only main difference is that we don't consider the same grids to compute the two dimensional integral over $\left[0, T_{1}\right] \times\left[0, T_{2}\right]$ and to discretize the condition $\mathbb{1}_{X(s)<X(t), \forall s \triangleleft t}$. As a matter of fact, this last discretization is time consuming and can be faster performed on a coarser grid without impacting the precision.

More precisely, let

$$
G_{n_{1}, n_{2}}\left(T_{1}, T_{2}\right)=\left\{\left(t_{i}^{1}, t_{j}^{2}\right) ; t_{i}^{1}=\frac{i}{n_{1}} T_{1}, t_{j}^{2}=\frac{j}{n_{2}} T_{2}, 0 \leq i \leq n_{1}, 0 \leq j \leq n_{2}\right\}
$$

be the grid for the trapezoidal rule and let $m_{1}$ dividing $n_{1}$ and $m_{2}$ dividing $n_{2}$, then the subgrid of $G_{n_{1}, n_{2}}\left(T_{1}, T_{2}\right)$ defined for the discretization of the condition

$$
X(s)<X(t), \forall s \triangleleft t
$$


is

$D_{m_{1}, m_{2}}\left(T_{1}, T_{2}\right)=\left\{\left(s_{k}^{1}, s_{l}^{2}\right) ; s_{k}^{1}=\frac{k}{m_{1}} T_{1}, s_{l}^{2}=\frac{l}{m_{2}} T_{2}, 0 \leq k \leq m_{1}, 0 \leq l \leq m_{2}\right\}$.

In particular, for each point $t$ of $G_{n_{1}, n_{2}}\left(T_{1}, T_{2}\right)$, we define the past of $t$ (with respect to the order $\triangleleft)$ as the set

$$
P_{t}=\{s ; s \triangleleft t\} \cap D_{m_{1}, m_{2}}\left(T_{1}, T_{2}\right)
$$

in order to construct our numerical routine MAXNSGP2 similarly to MAXNSGP. To do so, we consider the one dimensional algorithm of Section 3.2.1 on the vectors

- $\left(X_{1}^{\prime}\left(t_{i}^{1}, 0\right), X\left(P_{\left(t_{i}^{1}, 0\right)}\right), X\left(t_{i}^{1}, 0\right)\right)_{0 \leq i \leq n_{1}}$ for $B_{1}$,

- $\left(X_{2}^{\prime}\left(0, t_{2}^{j}\right), X\left(P_{\left(0, t_{2}^{j}\right)}\right), X\left(0, t_{2}^{j}\right)\right)_{0 \leq j \leq n_{2}}$ for $B_{2}$,

- $\left(X_{2}^{\prime}\left(T_{1}, t_{2}^{j}\right), X\left(P_{\left(T_{1}, t_{2}^{j}\right)}\right), X\left(T_{1}, t_{2}^{j}\right)\right)_{0 \leq j \leq n_{2}}$ for $B_{3}$,

while the computation of the interior is based on a trapezoidal rule on the grid $G_{n_{1}, n_{2}}\left(T_{1}, T_{2}\right)$ combined with the previous algorithm on the vector

$$
\left(X_{1}^{\prime}\left(t_{i}^{1}, t_{j}^{2}\right), X_{2}^{\prime}\left(t_{i}^{1}, t_{j}^{2}\right), X_{11}^{\prime \prime}\left(t_{i}^{1}, t_{j}^{2}\right), X\left(P_{\left(t_{i}^{1}, t_{j}^{2}\right)}\right), X\left(t_{i}^{1}, t_{j}^{2}\right)\right)_{0 \leq i \leq n_{1}, 0 \leq j \leq n_{2}} .
$$

An exemple of application of MAXNSGP2, available at Mourareau (2015), is given in Appendix B. Finally, note that MAGP by Mercadier (2005, 2006) is another version that uses the WAFO toolbox and that does not give an estimation of the error. As far as we can tell, MAGP was until recently the only program that concerns two-dimensional stationary Gaussian fields.

3.2.3. A program for the Poisson method. Finally, the Poisson approximation is illustrated by functions pPOISSON and qPOISSON, that can be found at Mourareau (2015). An example of utilization is given by

$$
\mathrm{p}=\text { qPoisson }(0.05,10,1)
$$

where $\alpha=0.05$ is the level fixed by the user, $T=10$ is the length of the interval and $\rho^{\prime \prime}(0)=-1$.

\section{Applications to Statistics}

In this section we want to consider the three classical approximations (with their variants) given in introduction and the Poisson approximation of Section 2.3. Our aim is to compare these tools in various scenarios.

4.1. Simulation procedure. Our computation scheme is the following. For a given size of interval (or rectangle), we use the relevant approximation of Section 2 to compute a $\alpha$ approximate threshold $u_{\alpha}$ and then we use the relevant numerical program to compute the corresponding actual level $\bar{\alpha}$. The approximation is said satisfactory if there is a small discrepancy between $\alpha$ and $\bar{\alpha}$.

Such a kind of study has been performed in the numerical study of the domain of validity of the classical $\chi^{2}$ test. Indeed, the well-known condition "the expected size of each cell must be greater than 5 " corresponds to conditions that ensures 
that the actual level $\bar{\alpha}$ differs from the nominal level $\alpha$ by less than $20 \%$ in relative variation (see Greenwood and Nikulin, 1996). In other words, for a nominal level $\alpha$ of $5 \%$ (resp 1\%) we demand the actual level to be in $(4 \%, 6 \%)$ (resp. $(0.8 \%, 1.2 \%)$ ).

In the interpretation of our numerical experiments we will generally consider a more restrictive rule of relative variation of $5 \%$ instead of $20 \%$. In ther words we will demand, for a nominal level of $5 \%$, (resp. 1\%) the true level to belong to $[4.75 \%, 5.25 \%]$ (resp. $[0.95 \%, 1.05 \%]$.

\subsection{The processes.}

For the Pickands approximation,

- the stationary Gaussian process with covariance $\rho(t)=\exp \left(-t^{2} / 2\right)$ for which the result is known from the MGP program,

- the stationary Gaussian process with covariance $\rho(t)=\exp \left(-t^{1.95}\right)$ for which the result is approximatively known from the lower bound of the MGP program.

- the Ornstein-Uhlenbeck process with covariance $\rho(t)=\exp (-|t|)$ for which the result is exactly known from the pou function.

These are the few case where we are able to perform a numerical study for $\beta$ processes.

For the Rice and the Poisson approximation, we consider three stationary processes with covariance

- $\rho(t)=\exp \left(-t^{2} / 2\right)$,

- $\rho(t)=1 / \cosh (t)$,

- $\rho(t)=\operatorname{sinc}(\sqrt{3} t)=\frac{\sin (\sqrt{3} t)}{\sqrt{3} t}$.

For the Euler characteristic, we consider isotropic and stationary Gaussian fields with covariances

- $\rho(t)=\exp \left(-\|t\|^{2} / 2\right)$,

- $\rho(t)=(1+\|t\|) \exp (-\|t\|)$ which is a Matern covariance with parameter $3 / 2$.

To give a precise meaning to the interpretation, we will always normalize our processes with $\rho(0)=1$ and "unit speed transformation" $-\rho^{\prime \prime}(0)=1$.

4.3. General presentation of the figures. In a first part we consider the problem of determination of a threshold for test of level $\alpha=0.01$ and $\alpha=0.05$. In the second part we consider the problem of the determination of the median $\alpha=0.5$.

Let us consider the example of Figure 4.3, the others being very similar. As a function of the length $T$ of the set, in abscissa, a threshold $u$ has been computed using the Rice bound given by (2.4) for $\alpha=0.05$. This bound is only approximative. So the true level is not really $\alpha$ and is measured using a numerical tool here MGP. Recall that the upper bound is in general closer to the reality. From left to right, the three processes of Section 4.2 are considered.

\subsection{Results and comments. We give a list of the main points}

- Figures 4.3 and 4.4 show that the Rice method is, in general, satisfactory for statistical application: the upper-bound in red which is the more precise is very close to the nominal value. 
- Figures 4.5, 4.6 and 4.7 show that the form (2.2) or (2.3) of the Pickands methods perform well for large time intervals but neither is preferable to the other in the general case. Moreover, for small intervals, both give too low levels. If the heruristic correction for small intervals is performed, the method performs well in every conditions. Note that there is some instability for very small intervals which is studied Figure 4.8: a "zoom" of Figure 4.5. It shows that the local increase around $T=0$ of the actual level is not that important.

- Both Rice (Figure 4.9) and Pickands (not displayed) methods are not relevant to compute the median as soon as $T$ is not very small.

- In case of smooth processes, the Poisson method performs well in every situation.

- The problem of computing the median of $M_{T}$ for $\beta$ processes $\beta \neq 2$ remains open.

- The Euler characteristic method performs rather well, except for $\alpha=0.5$ and very small rectangles. In our interpretation this is due to the fact that in almost every considered case the level $u$ is high.

4.4.1. $1 \%$ and $5 \%$ critical values.
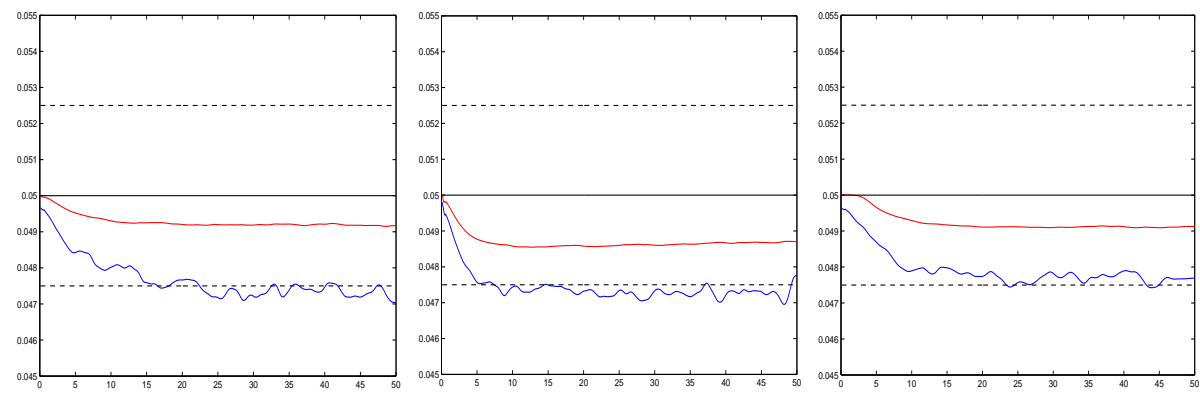

Figure 4.3. Exact level of a test constructed for a nominal level of $\alpha=\mathbf{5} \%$ using the Rice method (see Section 2). An upper bound (red line) and a lower bound (blue line) of the level are computed by the program MGP (see Section 3.1.1). From left to right, the processes considered have covariance function $\rho(t)=\exp \left(-t^{2} / 2\right)$, $\rho(t)=1 / \cosh (t)$ and $\rho(t)=\operatorname{sinc}(\sqrt{3} t)$.
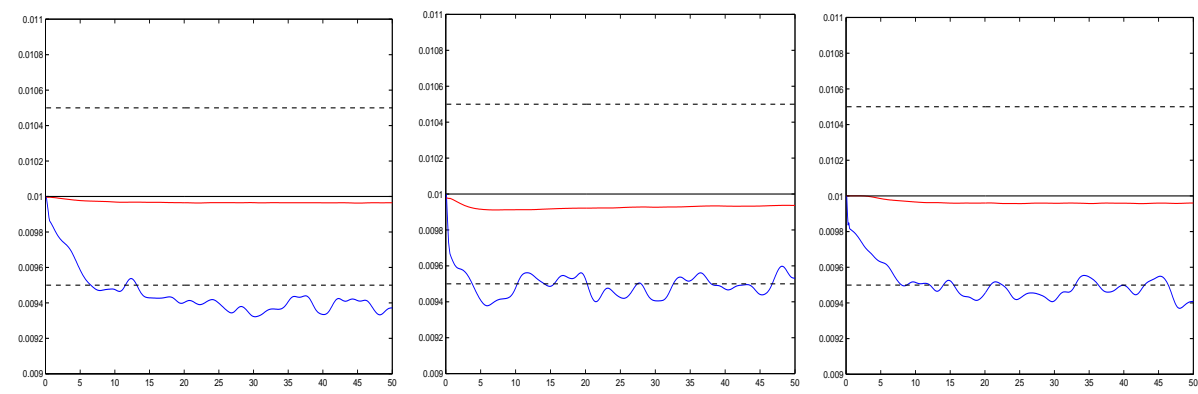

Figure 4.4. Same as Figure 4.3 except that $\alpha=0.01$. 

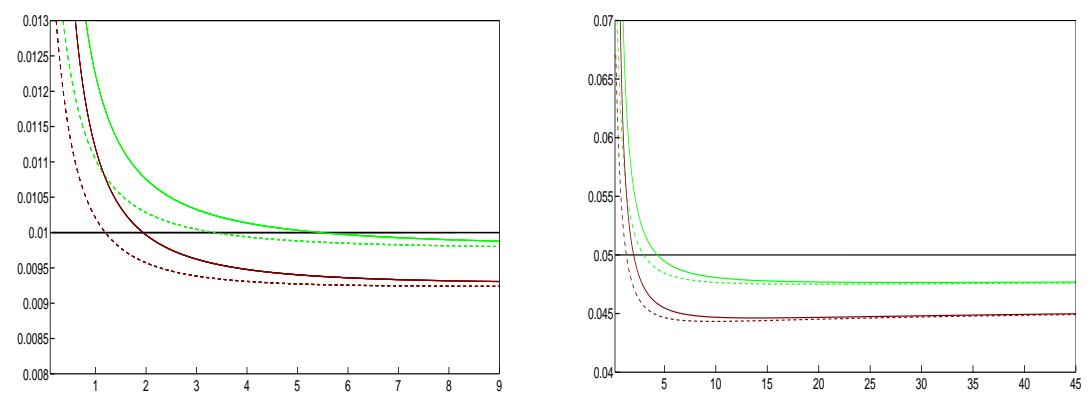

FiguRE 4.5. Exact level of a test constructed for the Ornstein-Ulhenbeck process and a nominal level of $\alpha=\mathbf{1} \%$ (left) or $\alpha=\mathbf{5} \%$ (right) using the Pickands method and the program pou. The level corresponding to equation (2.2) is given in brown, the one of equation (2.3) is given in green while the modified versions including $\bar{\Phi}(u)$ are given in dashed.
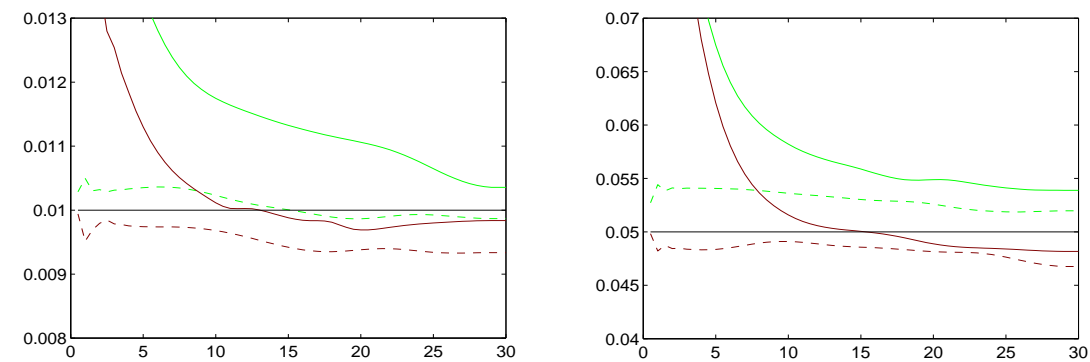

Figure 4.6. The process considered here has covariance $\rho(t)=\exp \left(-t^{1.95}\right)$; Exact level of a test constructed for a nominal level of $\alpha=\mathbf{1} \%$ (left) or $\alpha=\mathbf{5} \%$ (right) using the Pickands method. Our numerical computation uses the lower bound of MGP, thus it provides an under estimation. The meaning of the colours and dashed lin is the same as in Figure 4.5.
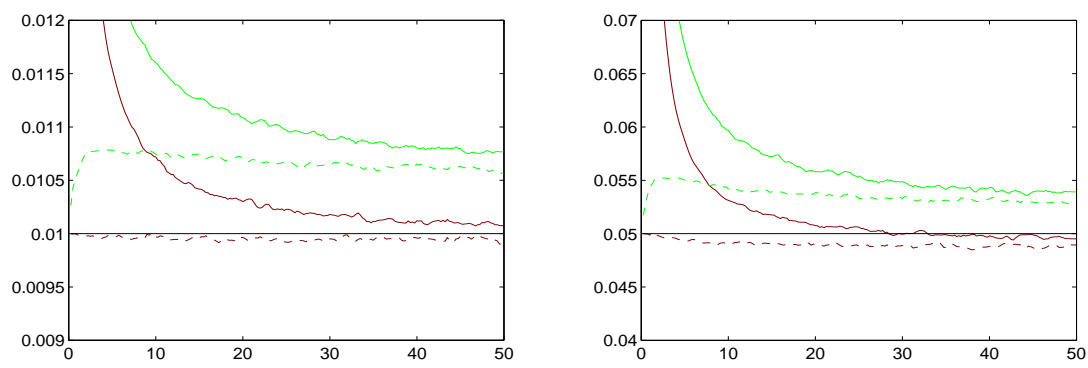

Figure 4.7. Same as Figure 4.6 except that (a) the covariance is now $\rho(t)=$ $\exp \left(-t^{2} / 2\right)$, (b) the computation uses the upper-bound of MGP which is known to be precise. 

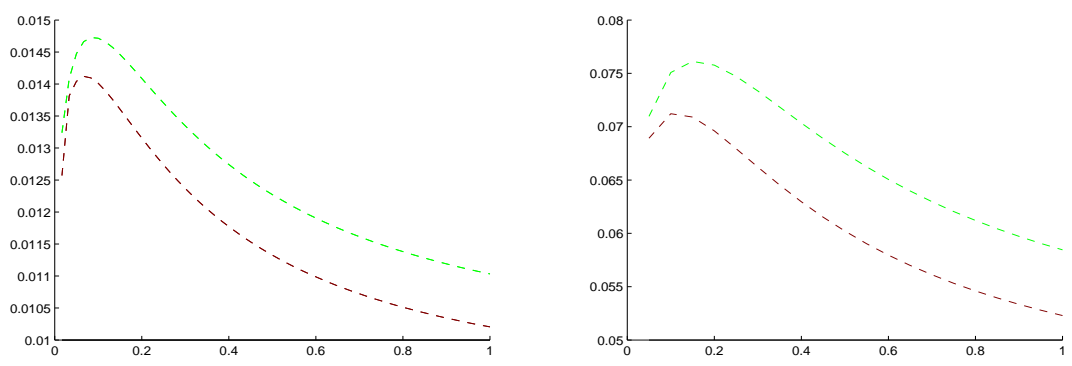

Figure 4.8. Zoom of Figure 4.5 for the dotted lines and small values of $\mathrm{T}$.

\subsubsection{Computing the median of $M_{T}$.}
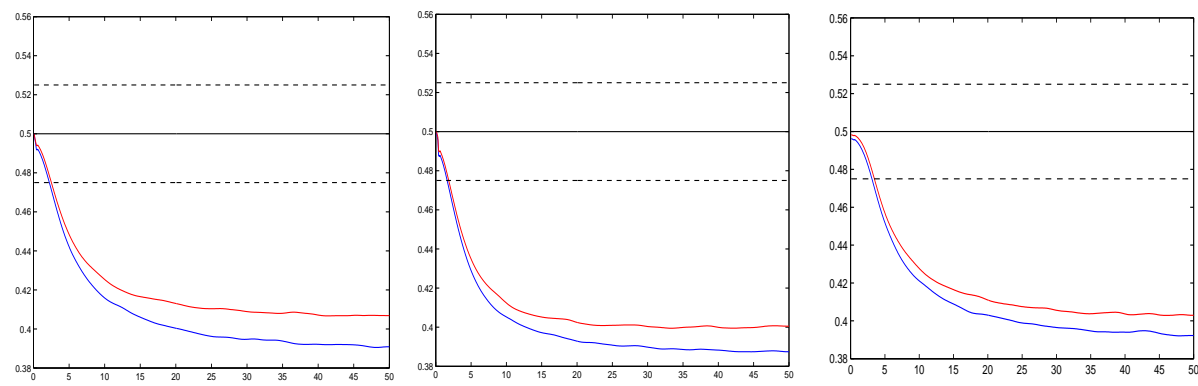

FiguRE 4.9. Exact level of a test constructed for a nominal level of $\alpha=\mathbf{5 0} \%$ using the Rice method (see Section 2). An upper bound (red line) and a lower bound (blue line) of the level are computed by the program MGP (see Section 3.1.1). From left to right, the processes considered have covariance function $\rho(t)=\exp \left(-t^{2} / 2\right)$, $\rho(t)=1 / \cosh (t)$ and $\rho(t)=\operatorname{sinc}(\sqrt{3} t)$.
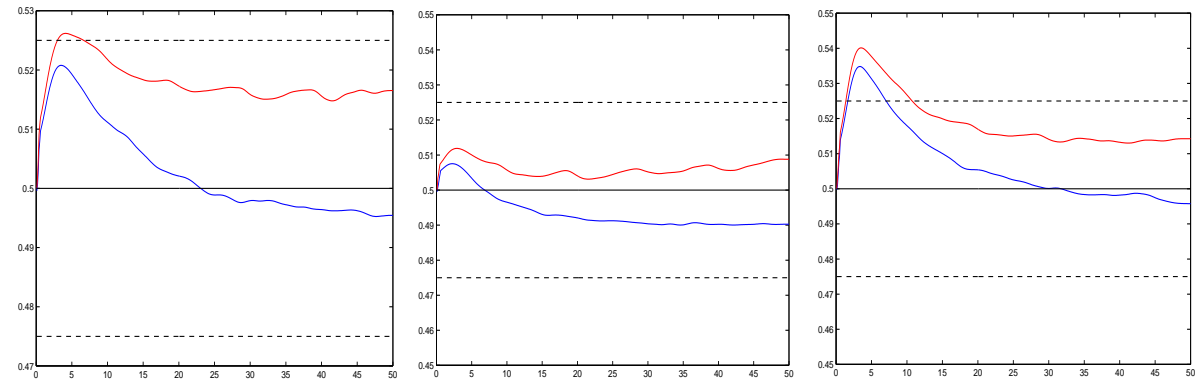

Figure 4.10. Exact probability of $M_{T}$ to be above the approximate median computed with the Poisson method (see Section 2). An upper bound (red line) and a lower bound (blue line) of the probability are computed by MGP (see Section 3.1.1). From left to right, the processes considered have covariance function $\rho(t)=\exp \left(-t^{2} / 2\right), \rho(t)=1 / \cosh (t)$ and $\rho(t)=\operatorname{sinc}(\sqrt{3} t)$. 


\subsubsection{Two dimensional case.}
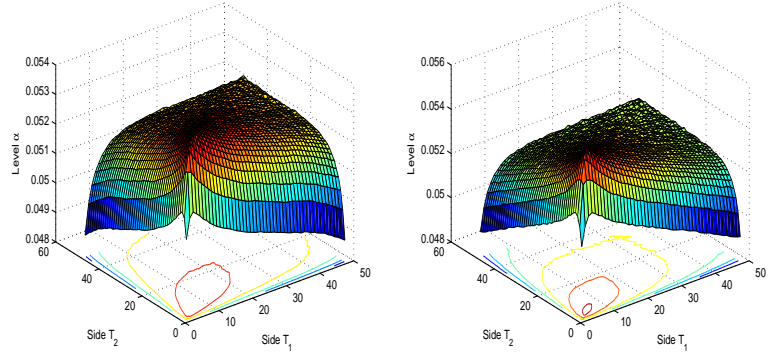

Figure 4.11. Exact level of a test constructed for a nominal level of $\alpha=\mathbf{5} \%$ using the Euler characteristic method (see Section 2) on subsets of $[0,50]^{2}$. The upper bound presented here is computed by the program MAXNSGP2 (see Section 3.1.1) and the processes considered have covariance function $\rho(t)=\exp \left(-t^{2} / 2\right)$ (left) and $\rho(t)=(1+\|t\|) \exp (-\|t\|)$ (right).
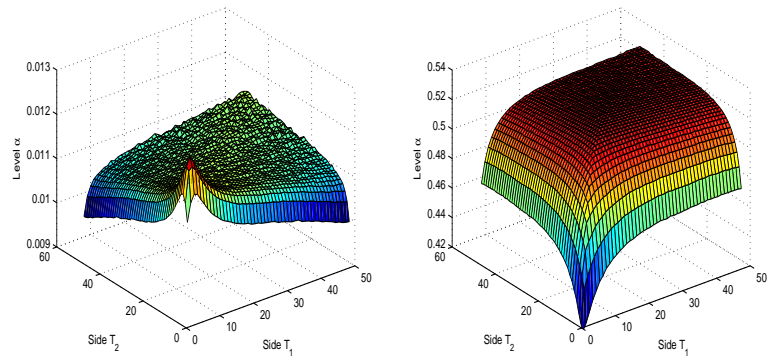

Figure 4.12. Same as Figure 4.11 except that : The covariance is always $\rho(t)=$ $\exp \left(-t^{2} / 2\right)$ and $\alpha=0.01$ (left) and $\alpha=0.5$ (right)
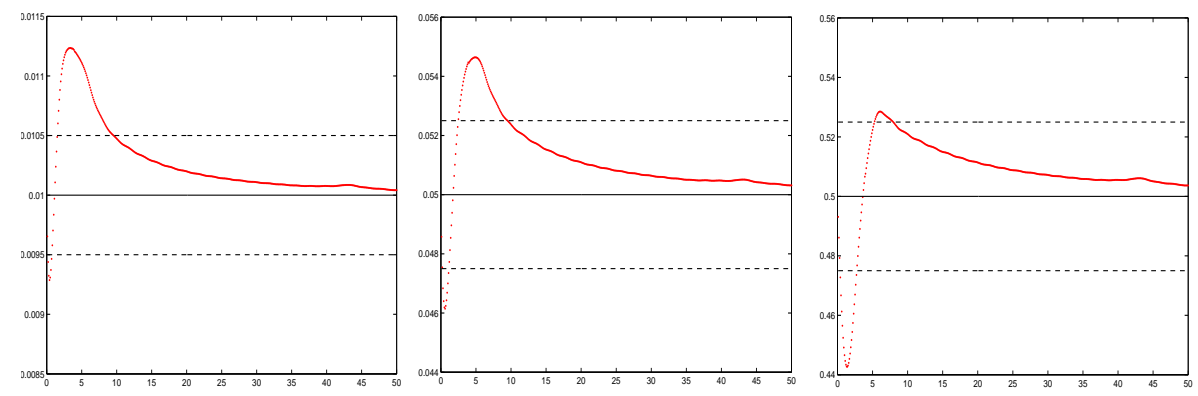

Figure 4.13. Exact level of a test constructed for a nominal level of, from left to right, $\alpha=\mathbf{1} \%, \alpha=\mathbf{5} \%$ and $\alpha=\mathbf{5 0} \%$, using the Euler characteristic method (see Section 2) on subsets of the diagonal of $[0,50]^{2}$. The upper bound presented here is computed by the program MAXNSGP2 (see Section 3.1.1) and the process considered has covariance function $\rho(t)=\exp \left(-t^{2} / 2\right)$. 


\section{Conclusion}

The present paper has shown that the classical bounds are efficient for statistical application as soon as the good version of Pickands method is used. However, they are not efficient for computing the median of the maximum. In that case, the proposed Poisson method performs well and is equivalent to the Rice method in the previous situations. In addition, Figure 5.14 show that it performs well for the computation of others fractiles. Finally, the Euler characteristic method is efficient in almost every cases.
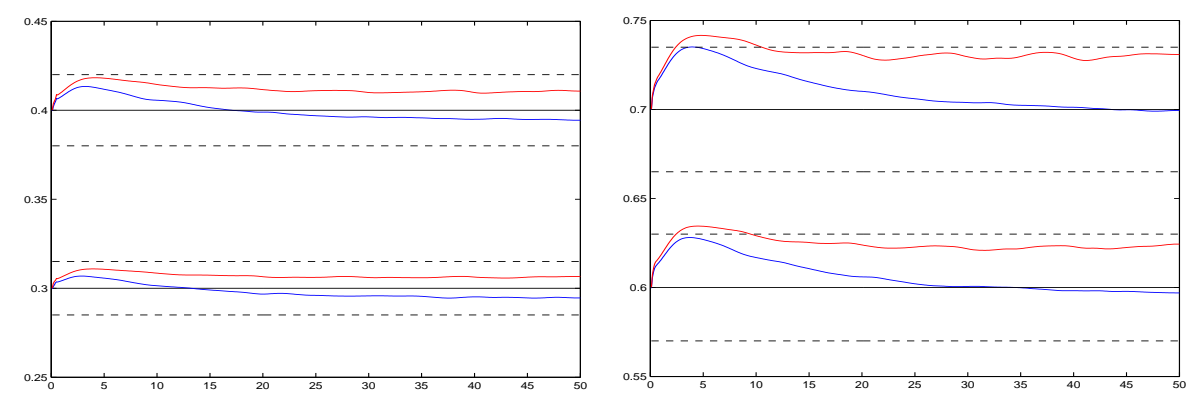

FIGURE 5.14. Exact probability of $M_{T}$ to be above the approximate $1-0.40$ (left top); $1-0.70$ (right top) $1-0.30$ (left bottom) $1-0.60$ (right bottom) fractile computed with the Poisson method (see Section 2) on subsets of $[0,50]$. An upper bound (red line) and a lower bound (blue line) of the level are computed by the program MGP (see Section 3.1.1). The process considered has covariance function $\rho(t)=\exp \left(-t^{2} / 2\right)$.

\section{Appendix A. MAXNSGP}

The syntax of MGP and MAXNSGP are very similar. For instance, to compute the probability that the maximum of the Gaussian process satisfying $m(t)=4$ and $r(s, t)=\frac{e^{s t}-1}{\sqrt{e^{s^{2}}-1} \sqrt{e^{t^{2}}-1}}$ exceed the level $u=1$ on the set $[1,3]$, it reads as

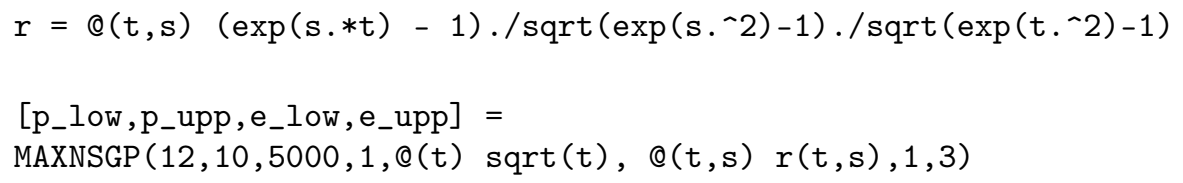

where

- 12 is the typical value for the number of Monte-Carlo (MC) replications in the MCQMC integration;

- 10 is the number of discretization point of the interval [1,3];

- 5000 is the number of points used for the QMC routine;

- 1 is the level of comparison;

- @(t) sqrt(t) is the mean function of the process;

- $@(t, s) r(t, s)$ is the covariance function of the process;

- 1 is the beginning of the interval;

- 3 is the end of the interval. 
Note that this kind of processes appear in the asymptotic theory of Likelihood Ratio Test in the case of a mixture, see Delmas (2003) for more details. In case of discrepancy, the upper-bound is, in general, more precise. Finally, the upper-bound is very efficient even for small values of $n$.

\section{Appendix B. MAXNSGP2}

Again, the syntax is very similar to the one of MGP and MAXNSGP. For instance, to compute the probability that the maximum of the Gaussian field satisfying $m(t)=0$ and $\forall(s, t) \in\left(\mathbb{R}^{2}\right)^{2}, r(s, t)=\exp \left(-\|t-s\|_{2}^{2}\right)$ exceed the level $u=2$ on $[0,5] \times[0,10]$, it reads as

$$
\begin{aligned}
& r=@(a, b, c, d) \exp (-(a-c) \cdot \sim 2 / 2-(b-d) \cdot \sim 2 / 2) \\
& \text { [p_low,p_upp, e_low, e_upp] }= \\
& \text { MAXNSGP2 }(12,20,40,2,4,1000,2, @(t, s) 0, r, 5,10)
\end{aligned}
$$

where

- 12 is the typical value for the number of Monte-Carlo (MC) replications in the MCQMC integration;

- 20 is the number of discretization points for trapezoidal rule on the x-axis;

- 40 is the number of discretization points for trapezoidal rule on the y-axis;

- 2 is the number of points used for the record set on the x-axis;

- 4 is the number of points used for the record set on the y-axis;

- 1000 is the number of points used for the QMC routine;

- 2 is the level of comparison;

- @ $(\mathrm{t}, \mathrm{s}) 0$ is the mean function of the process;

- $\mathrm{r}$ is the covariance function of the process;

- 5 and 10 denote the length of each side of the rectangle, here $[0,5] \times[0,10]$;

Note again that in case of discrepancy, the upper-bound is, in general, more precise.

\section{Appendix C. Maxgpbox}

Maxgpbox is a collection of matlab programs available at the webpage https://www.math.univ-toulouse.fr/ azais/softwares.php that performs exact calculations for the few cases for which an exact formula is known. It includes in particular the pou function devoted to the Ornstein-Uhlenbeck process.

\section{References}

R. J. Adler. The geometry of random fields. John Wiley \& Sons, Ltd., Chichester (1981). ISBN 0-471-27844-0. MR611857.

R. J. Adler and J. E. Taylor. Random fields and geometry. Springer Monographs in Mathematics. Springer, New York (2007). ISBN 978-0-387-48112-8. MR2319516.

J. M. P. Albin and H. Choi. A new proof of an old result by Pickands. Electron. Commun. Probab. 15, 339-345 (2010). MR2685014.

D. Aldous. Probability approximations via the Poisson clumping heuristic, volume 77 of Applied Mathematical Sciences. Springer-Verlag, New York (1989). ISBN 0-387-96899-7. MR969362. 
J.-M. Azaïs and C. Delmas. Asymptotic expansions for the distribution of the maximum of Gaussian random fields. Extremes 5 (2), 181-212 (2003) (2002). MR1965978.

J.-M. Azaïs and Alan Genz. Computation of the distribution of the maximum of stationary Gaussian processes. Methodol. Comput. Appl. Probab. 15 (4), 969-985 (2013). MR3117635.

J.-M. Azaïs and L.-V. Lozada-Chang. A toolbox on the distribution of the maximum of Gaussian process (2013). URL: https://hal.archives-ouvertes.fr/hal00784874/document.

J.-M. Azaïs and M. Wschebor. Level sets and extrema of random processes and fields. John Wiley \& Sons, Inc., Hoboken, NJ (2009). ISBN 978-0-470-40933-6. MR2478201.

N. A. C. Cressie. Statistics for spatial data. Wiley Classics Library. John Wiley \& Sons, Inc., New York, revised edition (2015). ISBN 978-1-119-11461-1. MR3559472.

K. Dȩbicki, E. Hashorva and L. Ji. Extremes of a class of nonhomogeneous Gaussian random fields. Ann. Probab. 44 (2), 984-1012 (2016). MR3474465.

C. Delmas. On likelihood ratio tests in Gaussian mixture models. Sankhyā 65 (3), 513-531 (2003). MR2060605.

A. B. Dieker and B. Yakir. On asymptotic constants in the theory of extremes for Gaussian processes. Bernoulli 20 (3), 1600-1619 (2014). MR3217455.

A. Genz. Numerical computation of multivariate normal probabilities. Journal of computational and graphical statistics 1 (2), 141-149 (1992). DOI: 10.1080/10618600.1992.10477010.

P. E. Greenwood and M. S. Nikulin. A guide to chi-squared testing. Wiley Series in Probability and Statistics: Applied Probability and Statistics. John Wiley \& Sons, Inc., New York (1996). ISBN 0-471-55779-X. MR1379800.

A. J. Harper. Pickands' constant $H_{\alpha}$ does not equal $1 / \Gamma(1 / \alpha)$, for small $\alpha$. Bernoulli 23 (1), 582-602 (2017). MR3556785.

M. R. Leadbetter, G. Lindgren and H. Rootzén. Extremes and related properties of random sequences and processes. Springer Series in Statistics. Springer-Verlag, New York-Berlin (1983). ISBN 0-387-90731-9. MR691492.

X. Li and G. Xu. Uniformly efficient simulation for extremes of Gaussian random fields. J. Appl. Probab. 55 (1), 157-178 (2018). MR3780388.

C. Mercadier. Magp toolbox (2005). URL: http://math.univ-lyon1.fr/ $\sim$ mercadier/.

C. Mercadier. Numerical bounds for the distributions of the maxima of some oneand two-parameter Gaussian processes. Adv. in Appl. Probab. 38 (1), 149-170 (2006). MR2213969.

Z. Michna. Remarks on Pickands' theorem. Probab. Math. Statist. 37 (2), 373-393 (2017). MR3745392.

S. Mourareau. http://mourareau.stephane.free.fr/Software.html (2015).

J. Pickands III. Asymptotic properties of the maximum in a stationary Gaussian process. Trans. Amer. Math. Soc. 145, 75-86 (1969). MR0250368.

V. I. Piterbarg. Asymptotic methods in the theory of Gaussian processes and fields, volume 148 of Translations of Mathematical Monographs. American Mathematical Society, Providence, RI (1996). ISBN 0-8218-0423-5. MR1361884.

S. O. Rice. Mathematical analysis of random noise. Bell System Tech. J. 23, 282-332 (1944). MR0010932. 
V. A. Volkonskiǐ and Y. A. Rozanov. Some limit theorems for random functions. I. Theor. Probability Appl. 4, 178-197 (1959). MR0121856.

K. J. Worsley, S. Marrett, P. Neelin, A. C. Vandal, K. J. Friston and A. C. Evans. A unified statistical approach for determining significant signals in images of cerebral activation. Human Brain Mapping 4 (1), 58-73 (1996). DOI: 10.1002/(SICI)1097-0193(1996)4:1<58::AID-HBM4>3.0.CO;2-O. 